\title{
Cooperativismo como una herramienta para el turismo de base comunitaria. La respuesta desde la literatura
}

\author{
Eduardo Mata Arratia* Yanelli Daniela Palmas Castrejón*** \\ Andrea Edurne Jiménez Ruíz**** Rocío del Carmen Serrano Barquín****** \\ Universidad Autónoma del Estado de México (México)
}

\begin{abstract}
Resumen: Este artículo aborda al desarrollo local desde la literatura científica y su vinculación con la actividad turística, siendo un beneficio adherente a la misma; sin embargo, la falta de un proceso claro de desarrollo, trae consigo un crecimiento insustentable. Aunado a ello, el desarrollo del sector rural ha convergido con la emancipación de las comunidades que buscan nuevas formas de mejorar su calidad de vida, una de ellas es el turismo, bajo la visión de "comunitario". Por lo que, se planteó como objetivo vislumbrar el estado del arte del turismo comunitario, el desarrollo local y el cooperativismo para conocer cómo se ha dado a través del tiempo la relación de estos tres conceptos, lo cual se realizó a partir de un análisis holístico de 137 artículos científicos en español; obteniendo como principales aportaciones, que existe una relación entre formas organizacionales y emprendimientos apegados al cooperativismo, entablando una estrecha relación con el turismo, lo que puede llevar a conseguir un desarrollo sustentable. Además, se tiene que el turismo comunitario debería considerar al cooperativismo como factor clave para el desarrollo local, ya que promueve la integración comunitaria, la gobernanza y el empoderamiento del territorio.
\end{abstract}

Palabras Clave: Desarrollo local; Turismo; Comunidad; Cooperativismo.

\section{Cooperativism as a tool for Community Based Tourism. The answer from the literature.}

Abstract: This article deals with local development as seen in the specialist bibliography and its links with tourism as an adherent benefit. Lack of a clear clear development process, it has been found brings unsustainable growth. In addition to this, the development of the rural sector has converged with the emancipation of communities seeking new ways to improve their quality of life, one of which is community tourism. Therefore, the objective was to chart the state of the art of community tourism, local development and cooperativism over time to see how these three concepts have evolved, using a holistic analysis of 137 scientific articles in Spanish. The main results of the study were that cooperative organisations in tourism can lead to achieving sustainable development. Therefore, community tourism should consider cooperativism as a key factor for local development, since it promotes community integration, governance and empowerment of the territory.

Keywords: Local development; Comunity; Tourism; Cooperativism.

"La pobreza no la crea la gente pobre. Ésta es producto del sistema que hemos creado, por ende, hay que cambiar los modelos y conceptos rígidos de nuestra sociedad"

Muhammad Yunus (2006)

\section{Introducción}

La globalización es un efecto multidimensional causado por la política económica dominante, que tiene sus inicios con la culminación de la Segunda Guerra Mundial, en donde se crearon grandes bloques

\footnotetext{
* Universidad Autónoma del Estado de México (México); E-mail: lalomata1995@gmail.com; https://orcid.org/0000-0002-3472-116X

* Universidad Autónoma del Estado de México (México); E-mail: hashir04@hotmail.com; http://orcid.org/0000-0002-7158-0212

*** Universidad Autónoma del Estado de México (México); E-mail: andreaedurnejr@gmail.com; https://orcid.org/0000-0002-6928-6567

***** Universidad Autónoma del Estado de México (México); E-mail: rocioserba@yahoo.com; https://orcid.org/0000-0002-5695-751X
} 
económicos que se fortalecieron a partir de alianzas bélicas en la mayor parte de los países involucrados, quienes obtuvieron la victoria en dicho conflicto (Herrera, 1997; Castro- Gómez, 1998; Prieto, 2002). Como consecuencia de la globalización se amplían los canales de venta, así como crecen los grandes capitales privados, surgen las metrópolis a nivel mundial, avanza la ciencia y la tecnología, y con ello empiezan algunos efectos socioculturales, como la producción masiva, migración del campo a las ciudades, aculturación de los pueblos, pérdida de identidad y tradiciones, entre otros (Bolívar, 2001; Mateus y Brasset, 2002; Martín-Barbero, 2003).

Además de los efectos socioculturales, se notó crecientemente la afectación a los recursos naturales debido, entre otras cosas, a la producción en masa, sobrepoblación, depredación múltiple de ecosistemas y extinción de especies de flora y fauna, calentamiento global y muchos más. Todos los problemas antes mencionados por lo regular, involucran en mayor medida a los países industrializados, que regularmente son los encargados del orden global, siendo entonces, los efectos de su industria los que recaen en los países en vías de desarrollo. (Reyes, 2001; Artaraz, 2002; Alburquerque, 2003).

Los efectos negativos de las grandes potencias hacia los países en vías de desarrollo, bajo el paradigma de progreso (Barkin, 2001; Alburquerque; 2008; Andrade, Marinho y Lima, 2017), sólo limitan considerable-mente su desarrollo integral, y luchan por lograr inversión trasnacional y aumentar su Producto Interno Bruto (PIB) y otros indicadores, pero únicamente han traído una marcada desigualdad social, carente de oportunidades para mejorar la calidad de vida, inseguridad alimentaria, deficiencia en la educación y la salud (Rubio, 2002; Tello, 2006), efectos que se ven con mayor énfasis en las comunidades rurales.

Tanto en los países desarrollados, como en los que están en la periferia, existen comunidades rurales, donde se concentran las actividades económicas primarias primordialmente, aunque actualmente hay un incremento en el sector terciario, como es el caso de los servicios y el turismo (Klein, 2005; Vázquez Barquero, 2007; Alburquerque, 2008). Algunas comunidades han buscado la forma de competir dentro del mundo globalizado; sin embargo, han sido pocas las que logran hacerlo considerando los principios del desarrollo local.

Por lo que, de la triada de conceptos de turismo comunitario, desarrollo local y el cooperativismo (Imagen 1), se busca que, por medio de un proceso holístico, estos conceptos se vuelvan prácticos, aspecto que puede lograrse si desde el análisis de los conceptos se hace holísticamente. Ya que para Briceño et al. (2010), "los fenómenos y sucesos son contemplados desde una perspectiva dinámica e interconectada tanto en el tiempo como en el espacio" (p.75), tal es el caso del concepto de desarrollo local que evolucionó dinámicamente, de ser esencialmente económico a involucrar aspectos relacionados con la sustentabilidad (Boisier, 2005; Alcañiz, 2008; Guzón Camporredondo, 2015). También incluye la reducción de impactos de actividades agresivas que se realizan sobre el ambiente, además de regenerar y restaurar aquellos que han sufrido estragos por la mano del hombre (Serrano-Barquín, 2006). Con el paso del tiempo el concepto se ha fortalecido buscando que este tipo de desarrollo se base en una economía verde, que los consumos sean racionales, y no de despilfarro, para permitir que se reduzcan los desechos que se generan y el consumo de materia prima, pero principalmente lograr el bienestar social (Palmas, Serrano- Barquín y Osorio, 2014).

Imagen 1: Análisis de conceptos por medio de un proceso holístico

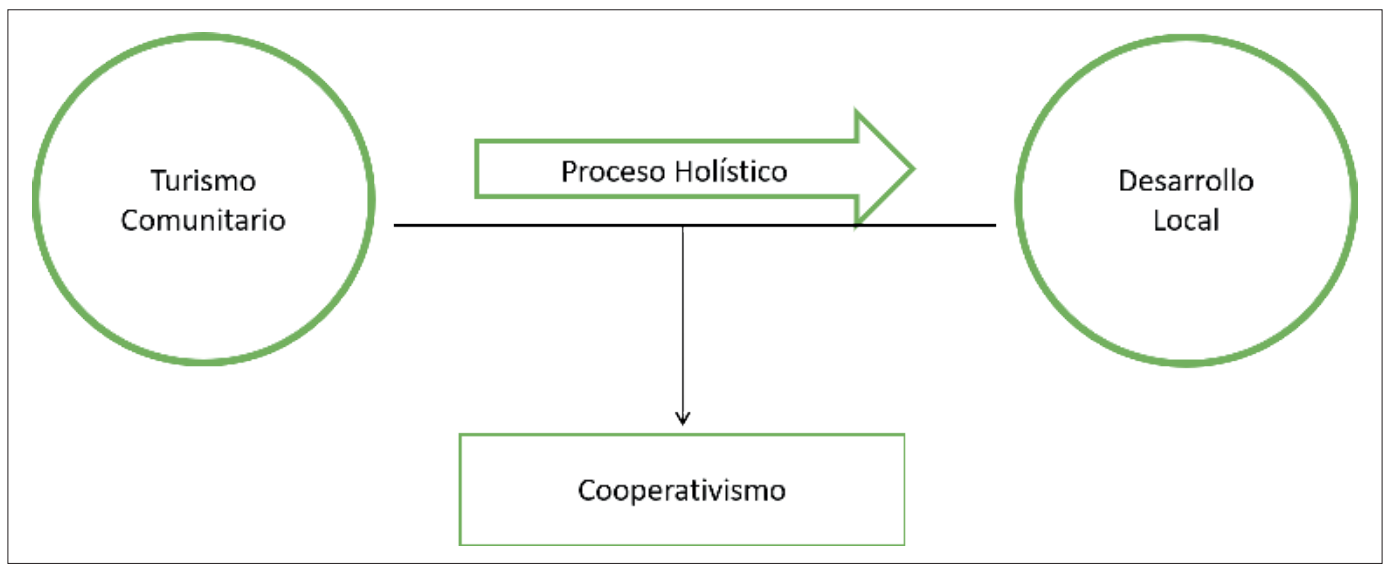

Fuente: Elaboración propia. 
Entre las estrategias de desarrollo local, se encuentra el turismo para comunidades que cuentan en su territorio con atractivos naturales, culturales, sociales o mixtos (Sharpley, 2000; Binns y Nel, 2002; Borma, 2015). A lo largo del tiempo se han realizado diversos proyectos turísticos en múltiples espacios, desafortunadamente no en todos los casos se ha podido observar un beneficio integral comunal, pues en la mayor parte no se logra apreciar una mejora en la calidad de vida, pero se perciben impactos negativos en los recursos naturales, socioculturales, políticos y tecnológicos.

Algunos teóricos han propuesto, a partir de observar las deficiencias de algunos proyectos, al cooperativismo como una forma para poder alcanzar las metas del desarrollo local en un lapso corto e incluso es tomado como un componente esencial (Martínez, 1999; Caballero, 2015; Mata y Pascual, 2019). Lo cual, a escala global, ha funcionado en algunos países; sin embargo, aún es controversial y está en construcción.

Es por ello que se considera conveniente reflexionar en torno a los conceptos de desarrollo local, turismo comunitario y cooperativismo, con el objetivo de vislumbrar el estado del arte de estos tres conceptos para conocer como se ha dado la relación de estos tres conceptos a lo largo del tiempo. Para ello se revisaron 137 artículos científicos por medio de un análisis holístico, ya que este permite examinar los conceptos de manera individual, sin aislarlos, entenderlos desde el punto de vista de múltiples interacciones y entrelazarlos de manera compleja, lo que lleva a una visión sistémica de la relación de los tres conceptos en determinado tiempo y espacio, según haya sido el objeto, sujeto y territorio de estudio del artículo analizado (Briceño et al., 2010). Se consideraron solamente artículos en español para determinar los casos de cooperativismo en América Latina y saber el estado actual de lo que se está haciendo en esta región.

Entre los principales resultados se tiene que, en el proceso de desarrollo local, el indicador con mayor énfasis es el mejoramiento de la calidad de vida de la población, pese a que el mismo, no únicamente se limita a la medición numérica, sino integra un conjunto de cualidades. Además, el cooperativismo es un factor clave del turismo comunitario como herramienta de desarrollo local, ya que la actividad turística con base en la comunidad, promueve la integración comunitaria, la gobernanza y el empoderamiento del territorio.

Se concluye, entre otras cosas, que el turismo de base comunitaria es una herramienta de desarrollo local en algunas comunidades, ya que no solamente involucra ofrecer y vender un servicio, lugar o producto, sino también es un medio de cohesión social, educación, emancipación y promoción de una visión de sustentabilidad de los recursos

\section{Hacía un desarrollo local a través de la evolución de un concepto.}

Si bien, en la mayoría de las comunidades rurales, a nivel mundial, resalta la pobreza, como una condición que dificulta a quienes la integran para lograr el desarrollo; actualmente, se han buscado diversas estrategias para que estas puedan mejorar su calidad de vida. Entre algunas estrategias se encuentran la construcción de nuevos modelos que modifiquen y mejoren a las comunidades marginadas, nuevos paradigmas que permitan realmente un desarrollo local, críticas en contraposición a un crecimiento económico desmesurado, entre otras (Vázquez- Barquero, 2000; Barkin, 2001; Arocena, 2002: Orozco y Núñez, 2013; Juárez, 2013).

Desde la postura económica neoliberal, Quintero y Gallardo (2008) mencionan que el proceso de globalización se ha fortalecido a partir de la década de los setenta, cuyas principales características son la liberación de los mercados (financiero, cambiario, y de bienes y servicios), apertura económica y libre comercio, y se tiene como consecuencia la reducción del papel del Estado a una participación limitada de regulador en los asuntos económicos, procesos de privatización de empresas públicas, desregulaciones, entre otros, con la finalidad de garantizar la libre movilidad de los capitales y los bienes producidos.

Bajo la misma postura se critica al capitalismo enfocado en el crecimiento económico, los resultados que arrojan los países en general son de crecimiento, pero no se debe confundir el concepto de crecimiento con el de desarrollo (Di Prieto, 2001); ya que no necesariamente la repartición de los bienes es equitativa entre todos los actores involucrados (Sánchez y Prada, 2014; Andrade, Marinho y Lima, 2017).

A partir de los planteamientos críticos al crecimiento económico desde la visión neoliberal, surge la idea emancipadora del desarrollo desde el punto de vista histórico. Boisier (1999), plantea acerca de la evolución del término haciendo énfasis en momentos históricos, como la culminación de la Segunda Guerra Mundial ya que, a partir de convenios o acuerdos generados por los países ganadores, algunas organizaciones mundiales propusieron metas u objetivos para alcanzar el desarrollo.

En sí, el desarrollo teóricamente se plantea como todos aquellos aspectos cualitativos bajo los cuales es posible mejorar la calidad de vida de las personas y que no siempre se pueden medir únicamente con base en datos cuantitativos, ampliando los parámetros hacia otros más de carácter subjetivo, como bienestar y el propio desarrollo humano como un fin del proceso (Álvarez y Alonso, 2006; Monge-Rodríguez 
y Macías- Chávez; 2016). Por lo que el desarrollo es visto como un proceso, cuya principal misión era dirigida a la obtención de riquezas, valores y alcances del crecimiento.

A partir de los postulados acerca del desarrollo, como nuevo enfoque en las agendas nacionales de los países, a mediados de los años noventa surgen nuevas posturas sobre el enfoque comunitario que se podrían incorporar al desarrollo y con ello nuevos autores que fundamentan el concepto, sin dejar de lado el ámbito económico como sugiere Vázquez-Barquero (2000), entran en debate los términos de exógeno y endógeno. Es así como empieza a surgir la visión del desarrollo local como una respuesta por parte de las pequeñas comunidades o regiones a los grandes capitales en las metrópolis más importantes, donde se ha pensado existe el progreso y se mitiga la pobreza.

Como concepto, en su etapa inicial, el desarrollo local es entendido únicamente como un proceso económico, según la Organización Internacional del Trabajo (OIT, S.f.; en Alburquerque, 2004; 7) "un proceso de desarrollo participativo que fomenta los acuerdos de colaboración entre los principales actores públicos y privados de un territorio, posibilitando el diseño y la puesta en práctica de una estrategia de desarrollo común a base de aprovechar los recursos".

El desarrollo local es entendido como un proceso en un territorio, en donde se unen las experiencias, recursos y capitales en beneficio de un espacio definido; además de buscar una mejor calidad de vida para las personas que viven en dicho espacio (Juárez, 2013). Cabe señalar que dentro de la ampliación del concepto de desarrollo se involucran términos como la sustentabilidad, la cual ha sido abordada por las organizaciones mundiales como la forma de desarrollo del presente, sin que comprometa los recursos para las generaciones venideras, tal como lo señala el Programa de Naciones Unidas para el Desarrollo (PNUD, 1990). Aunque la sustentabilidad es vista en mayor parte en el plano ecológico, también involucra los ámbitos económico, sociocultural, político y tecnológico.

Como parte de las críticas al desarrollo local, Boisier (1999) menciona que "es una utopía social por excelencia", además de expresar que el sentido de más acepción del desarrollo es verlo desde una perspectiva territorial, puesto que no trata de algo sustancial, sino únicamente de algo espacial. En contraparte, la propuesta de un desarrollo regional consiste en un cambio estructural localizado en una región o lugar en específico donde se busca el progreso (pp. 8). Finalmente contextualiza al desarrollo local como una cuestión popular, donde se prioriza al contenido; sin embargo, cabe señalar que no es apto para todos los casos dado lo intrínseco del proceso.

Otra postura crítica acerca del desarrollo local por Vázquez- Barquero (2007) plantea al desarrollo endógeno como una visión más cercana al ideal de los entornos comunitarios. El cambio más fuerte en la etapa evolutiva del desarrollo local se puede visualizar a partir de la preocupación ambiental, revalorización cultural, mejores prácticas políticas y mejoras tecnológicas, además del entorno económico que fue el principal motivo por el cual se buscó integrar a comunidades marginadas dentro de dicho proceso para poder competir dentro de la economía globalizadora.

El desarrollo local entonces se puede entender como un proceso de mejora a nivel regional, que mediante una visión sustentable busca el progreso integral de un espacio geográfico delimitado, siendo la diversificación de las actividades, el cooperativismo y el empoderamiento herramientas clave del proceso, a diferencia del crecimiento el desarrollo no tiene como principal indicador la acumulación de riqueza, es más bien cualitativo además de carácter emancipador contra el modelo de crecimiento neoliberal.

Ahora bien, el turismo es una actividad que se ha desarrollado con el paso de los años, los indicadores se han elevado exponencialmente a partir de la culminación de los conflictos bélicos mundiales, de la época de la modernización económica en los años 50's; factores como el crecimiento de capitales privados, liberación de los mercados, avances tecnológicos en los diferentes sistemas de transportes, entre otros han sido indicadores benéficos para la actividad turística. Al mismo tiempo, creció la modalidad conocida como turismo de masas, en su mayor parte practicada como "sol y playa" o también el creciente turismo cultural. (Vega y Vivas, 2007; Osorio, 2010; Alvarado y Martínez, 2013).

El turismo tomó relevancia a partir del contexto histórico de los avances tecnológicos que permitieron su progreso, eficiencia, así como la diversificación en cuanto a sus modalidades, es una actividad en constante desarrollo, suele ir innovando conforme a las tendencias y necesidades de las personas a nivel global. Al ser considerado también como un fenómeno sociológico, sufre cambios dinámicos conforme evoluciona la sociedad, pues en los últimos años se han incrementado las tipologías del turismo, así como las formas en las que se desarrolla.

Por mucho tiempo crecieron grandes destinos turísticos sin una adecuada planificación, ni responsabilidad social por parte de los gobiernos, residentes, turistas y empresas trasnacionales, tanto del sector turístico como de otros que obtienen ganancias de la actividad. Sin embargo, con el pasar de los años las comunidades sufrieron más perjuicios, a comparación de lo que recibían en ganancia porque se desarrollara el turismo en 
su territorio, el daño de la que por unos años se consideró como una alternativa ideal para la erradicación de la pobreza (Blázquez, Cañada y Murray, 2011; Lalangui, Espinoza y Pérez, 2017)

Los impactos negativos en la actividad turística en masa, llevaron a la confrontación acerca del daño notable que ocasionan los diversos proyectos de turismo, bajo la concepción de generación de riquezas a costa de grandes costos y daños irreparables; en varios ámbitos como la naturaleza, que presenta contaminación, pérdida de biodiversidad, o como los efectos en la economía, tales como la inflación o el encarecimiento del predio; en cuanto al ámbito sociocultural, la transculturación, aculturación, pérdida de sitios patrimonio y sobrecarga de los lugares públicos (Gruter, 2013; Picornell,2015)

A partir de los problemas generados por el turismo en masa, con apego a las agendas internacionales; donde se discutían problemas como el cambio climático, la pérdida del patrimonio cultural, entre otras, así como la tensión ocasionada en gran medida por la falta de planificación de los destinos. Surge la plataforma de turismo alternativo, modalidades pensadas en la reducción de los impactos masivos del turismo, desarrollo de actividades en sitios de naturaleza, inclusión de los miembros de las comunidades, entre otras. (Pearce, 1992; Jafari, 2005).

La tipología de turismo alternativo se desarrolló en los últimos treinta años, con el objetivo de reducir los impactos negativos ocasionados en los grandes destinos, causa de la no planificación, además de que la clasificación alternativa ofrece nuevas formas de hacer turismo, en entornos más naturales, cercanos a las comunidades, ofreciendo más que servicio una experiencia. De ahí que los conceptos de desarrollo local y turismo se relacionaran.

\section{Turismo de base comunitaria como alternativa de desarrollo local en América Latina.}

Como es bien sabido, el turismo debería contribuir a las comunidades receptoras bajo los tres pilares de la sustentabilidad (social, económico y ecológico) y siguiendo diversas acciones que van relacionadas con los objetivos o políticas planteadas por organizaciones mundiales como la Organización Mundial del Turismo (OMT). Por ejemplo, algunas acciones que han sido propuestas, son el uso controlado de los recursos naturales, preservación de especies, respeto y revalorización de las culturas locales, la repartición equitativa de las ganancias generadas por concepto de turismo, así como de actividades relacionadas y el mejoramiento de la calidad de vida. (López-Guzmán, Sánchez- Cañizares, 2009)

A partir de las diferentes modalidades de turismo alternativo, surge en los años 80's la visión del turismo comunitario o de base comunitaria, el cual consiste en apoyarse en la comunidad para desarrollar la actividad turística, que va desde involucrarse con la prestación de los servicios, hasta la propia organización de los mismos. Las comunidades suelen tener la iniciativa, emprenden y buscan un beneficio comunal para lograrlo, está también muy apegado al cuidado de los recursos naturales y bienes culturales. Dicho enfoque está abierto al entorno rural, aunque algunos teóricos lo enfocan más a un plano indígena. (Maldonado, 2005; López-Guzmán, Sánchez- Cañizares y Pavón, 2011; Burgos, 2016)

El turismo comunitario tiene fuertes vinculaciones sociológicas y antropológicas, intervienen los sistemas de relaciones sociales, el capital cultural, capacidad de organización al interior de la propia comunidad, la relación con el turista pues no todas las localidades se comportan de la misma forma con respecto a la llegada de turistas y la transformación de su sistema productivo, además de la forma de relacionarse con el mercado. Algunos estudios del turismo comunitario provienen principalmente de España, Perú, México y Ecuador (Milano, 2016).

Sin embargo, existen críticas hacia esta perspectiva de turismo, ya que por la propia naturaleza del sistema se puede caer en algunos vicios sociales como corrupción al interior de los grupos de trabajo, falta de liderazgo o dirección dentro del proyecto, la carencia en la autogestión de las comunidades, la aceptación de los residentes hacia la conducta de los visitantes (Goodwin y Santilli, 2006; De la Torre, 2010: Milano, 2016); lo interesante es vislumbrar que el cambio de paradigma es complejo, más aún entre personas de una zona rural, que una urbana o conurbada.

A pesar de las limitaciones del turismo comunitario, en su mayor parte es concebido como una alternativa de desarrollo local (Maldonado, 2005; Vázquez-De la Torre, López-Guzmán y Caridad; 2007; Ruíz et Al. 2008; Larco; 2015). En el caso de América Latina se presentó a partir de finales de los años noventa, la problemática central y las críticas se dieron porque los países intentaron copiar los modelos de turismo implementados con éxito en otros lugares (López-Guzmán, Sánchez- Cañizares y Pavón, 2011) y la fórmula no funciona de la misma manera, dado que las condiciones de cada lugar son propias y particulares.

A pesar de las críticas, pros y contras del turismo comunitario, en algunos países éste sigue funcionado, posicionándose como ejemplos de éxito, especialmente aprovechando los recursos naturales y culturales, 
es por ello que América Latina se ha convertido en terreno fértil para el desarrollo de proyectos turísticos emanados de las comunidades, especialmente las indígenas, como puede observarse en la imagen dos.

\section{Imagen 2: Casos de éxito de Turismo Comunitario en América Latina}

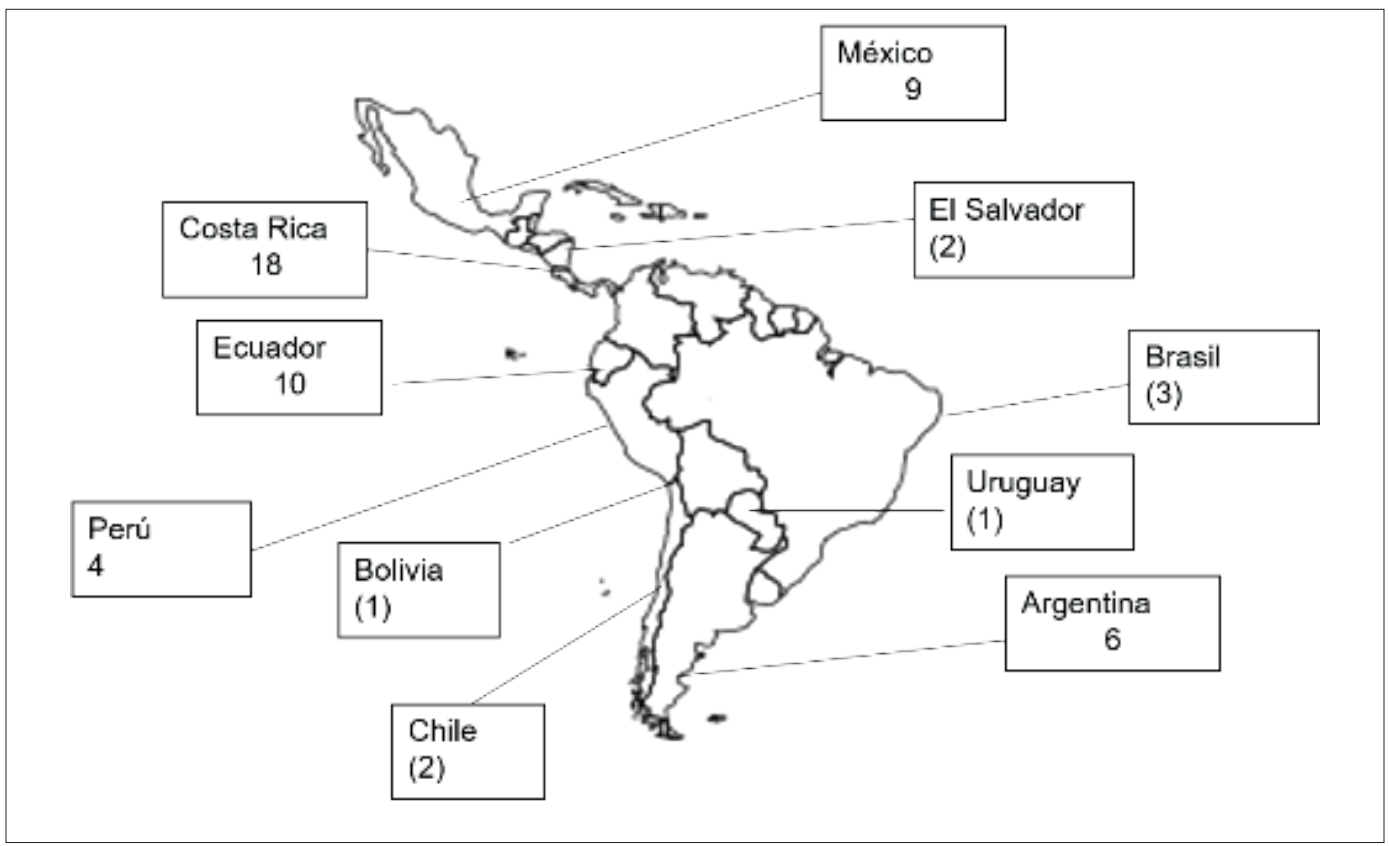

Fuente: Elaboración propia con base en Reportur, 2018; Palomino, Gasca, \& López, 2016; Mendoza, Figueroa, \& Godínez, 2015; Cañada, 2015; Maldonado, 2012; PNUD, 2012; Cardín y Álvarez C. 2012; Casas, Soler Domingo y Pastor, 2012; Díaz, 2010; Valle \& Cariño, 2009

Ecuador es uno de los países que se consideran líderes en turismo comunitario en América Latina, sus recursos naturales, culturales y mixtos, han servido para desarrollar la actividad turística, se considera que es una forma de entablar relaciones con las comunidades. Según aproximaciones teóricas en el país ecuatorial, se cuentan con alrededor de 250 emprendimientos de turismo, de las cuales 10 han sido consideradas como casos de éxito y analizadas en artículos científicos. Existe la Federación Plurinacional de Turismo Comunitario del Ecuador (FEPTC), organización encargada de este tipo de turismo, que propiamente lo ve como un instrumento estratégico de desarrollo multidimensional, atractivo para la demanda actual y complementa los modelos desarrollistas clásicos. Los proyectos registrados a través de la FEPTC son regionalizados y distribuidos en cinco redes de turismo geográficamente establecidas y es considerado por el Programa de las Naciones Unidas para el Desarrollo (PNUD, 2012) como un modelo de éxito. (Ruíz et. Al., 2008; Larco, 2015; Palacios 2016; Reyes, Ortega y Machado, 2017; Bravo y Zambrano, 2018).

En el caso del Perú, su Ministerio de Turismo le da el enfoque de "turismo rural comunitario", el cual define como "toda actividad turística que se desarrolla en el medio rural, de manera planificada y sostenible, basada en la participación de las poblaciones locales organizadas para beneficio de la comunidad, siendo la cultura rural componente clave del producto" (MINCETUR, 2015). Regiones como Arequipa, Cuzco y la región selva, destacan dentro de los proyectos de turismo comunitario, que la mayoría considera de éxito, han tomado la modalidad como parte de sus estrategias y planes nacionales de turismo. (Jaime, Casas y Domingo, 2011; Montoya, 2013).

En Chile, también hay presencia de estudios acerca del turismo comunitario, en donde destacan que esta modalidad se encuentra en las comunidades indígenas de dicho país como una estrategia para poder salir adelante, también ha servido para el rescate de recursos naturales, en el caso de los forestales donde se ha destacado que la base comunitaria ha cambiado su perspectiva acerca de los recursos con los que cuenta, así como de la forma en los que se puede dar un mejor uso. Otro punto de las indagatorias que se realizaron 
en Chile, fue la relación con el término "gobernanza", que en principio es la relación del sector público con el privado; sin embargo, la nueva visión complementa con el sector social que fue obviado anteriormente (Skewes, Henríquez, y Pilquiman, 2012; Pozas y Henríquez, 2013; Pacheco y Henríquez, 2016; Pilquiman, 2016).

Por otra parte, en la región de Norte y Centro América, destacan México y Costa Rica como destinos en los cuales se lleva a cabo el turismo comunitario. En Costa Rica, el propio gobierno ha reconocido en sus planes sectoriales de turismo dentro del Plan de Desarrollo Turístico 2002-2012 (ICT, 2006), donde la tipología del rural-comunitario ha sido destacada y se encuentra presente en una gran diversidad de emprendimientos turísticos del país. La Red de Alianza Conservacionista de Turismo Alternativo de Costa Rica (ACTUAR), creada en el año 2003 (Murillo-Soto, 2016), es un ejemplo mediante la revisión bibliográfica del caso de éxito, se pudo obtener dos perspectivas, una favorable en dónde los trabajadores son los dueños de los emprendimientos, procuran el manejo sustentable de los recursos, potencian el desarrollo endógeno (Andreu, 2008; Cardín y Álvarez, 2012). Por otra parte, se demostró que el ingreso obtenido por los emprendedores corresponde a un valor igual o menor que el salario mínimo; sin embargo, la mayoría de los emprendedores está convencido que al pertenecer a la Red incrementaron sus oportunidades (Murillo-Soto, 2016).

Ahora bien, hablando de México, en la Sierra Norte de Oaxaca, se presenta un caso de éxito conocido como "Pueblos mancomunados de Oaxaca", los cuales a partir de 1994 se han integrado en distintos emprendimientos de ecoturismo, siendo la comunidad "Benito Juárez" el pionero en la región, ofreciendo recorridos a las zonas forestales y hacia otras comunidades cercanas (Rosas -Baños y Correa-Holguín. 2016). Dentro del proceso de desarrollo que se ha ejecutado en la región por medio del ecoturismo durante más de veinte años, las claves del éxito han sido los procesos de gobernanza, así como el cooperativismo dentro de la unión de las comunidades para incluso denominarse como "mancomunados", empleando una red de cooperación a nivel regional, que lo ha posicionado como uno de los destinos más importantes de turismo comunitario en México (Palomino, Gasca y López, 2016).

Ambos destinos (México y Costa Rica), han destacado por sus recursos naturales mundialmente reconocidos, en dichos ejemplos se conjuntan conceptos ya mencionados y se suma la gobernanza (Trejos, 2009; Fernández, 2011; Palomino, Gasca y López, 2016). A través de este análisis, se puede deducir que la gran diversidad de recursos naturales y culturales con los que cuentan ambos países, han servido como factor de éxito en la implementación de modelos de turismo alternativo de base comunitaria; puesto que la finalidad de los proyectos comunitarios es que los trabajadores del producto sean los dueños.

\section{La influencia del cooperativismo para un turismo comunitario.}

Como se mencionó en un inicio, el avance de la globalización por todo el mundo en los últimos años, propone una jerarquización social basada en la adquisición de bienes, propiedades y nivel económico, por lo que surgieron grandes empresas de alcance intercontinental, las cuales se han desarrollado en países de primer mundo como también en los periféricos (Beck, Moreno y Borras, 1998; Pérez, Núñez y Font, 2016). Se promueve una cultura consumista, individualista y con ideal capitalista, no solamente en el sentido económico, sino también en el sociocultural, ya que históricamente el hombre se ha caracterizado por integrarse en sociedad para poder establecer condiciones de supervivencia.

Las cooperativas surgen como sociedades mercantiles con características especiales como la naturaleza de la empresa, que se conforma por socios o participantes locales, sin embargo, a diferencia de otra conformación empresarial las cooperativas se han distinguido por poder establecer vínculos o redes de cooperación entre sus miembros, lo que genera que en su mayor parte se vuelvan empresas resilientes, participativas, solidarias y comprometidas ya sea con sus socios o con una región propia. (Buendía-Martínez, 2014; Caballero, 2015)

Bajo la perspectiva de la literatura y pese a que las cooperativas nacieron formalmente bajo ese concepto en Europa en 1844, sociedades prehispánicas en América ya contaban con la visión estructural del cooperativismo, la organización y el propio sistema social, en México los aztecas implementaron el llamado "Capulli" como un ente cooperativo inmerso en la organización de dicha cultura prehispánica, pese a que se consideraba como una práctica consanguínea más que comunitaria, intervenía en las relaciones comunales, haciendo participativo y democrático la actividad económica.(Hernández, 2005; Andrade y Ochoa; 2017)

El turismo ha servido como un detonante económico para países en vías de desarrollo, los cuales cuentan con proyectos turísticos en gran medida apoyados por factores exógenos, desde la política gubernamental a nivel nacional, hasta la inversión en gran medida proveniente de grandes capitales trasnacionales, con la promesa de poder erradicar la pobreza, así como mejorar la calidad de vida de las comunidades receptoras de turismo, en donde se desarrollan grandes complejos turísticos (Orgaz, 2013; Toselli, 2015) 
Ahora bien, las empresas cooperativas que se encuentran inmersas en la actividad turística se han reconocido como un detonante del desarrollo sustentable, pues se considera que su naturaleza de cooperación y visión comunitaria puede establecer políticas internas de manejo de los recursos naturales, añadiendo que según algunas experiencias valoran los recursos propios de un lugar, promoviendo el cuidado de los ecosistemas y culturas (Maldonado, 2009; Maldonado y Maldonado, 2018; Reyes y Romano, 2019). Como se observa en la tabla 1, existen ventajas y desventajas a las que se enfrenta una comunidad en el momento de implementar una cooperativa.

\section{Tabla 1: Ventajas y desventajas de la implementación del cooperativismo como proceso inherente al turismo comunitario.}

\begin{tabular}{|c|c|}
\hline Ventajas & Desventajas \\
\hline $\begin{array}{l}\text { El cooperativismo promueve la organización } \\
\text { comunitaria, considerado como un ente solidario } \\
\text { en donde además de ver los beneficios económicos } \\
\text { también es posible lograr un desarrollo endógeno } \\
\text { a partir de la diversificación de actividades, así } \\
\text { como la asignación de tareas } \\
\text { - Los trabajadores no solamente son empleados } \\
\text { sino, al mismo tiempo son gestores y propietarios } \\
\text { de la sociedad, la responsabilidad, así como los } \\
\text { beneficios impactan de forma directa. } \\
\text { - Es una forma de organización y puesta en } \\
\text { marcha de proyectos comunitarios puesto que la } \\
\text { cooperación, no solo significa una empresa, sino } \\
\text { también puede ser funcional a manera de redes. } \\
\text { - En tiempos de catástrofes o eventos } \\
\text { excepcionales sirve como medio de apoyo y } \\
\text { mitigación de impactos. } \\
\text { - Son empresas netamente ligadas al territorio y } \\
\text { a la comunidad, siendo muchas veces casos de } \\
\text { impulso al desarrollo territorial. } \\
\text { Son fuente de empleo en lugares de alta } \\
\text { marginación social. }\end{array}$ & $\begin{array}{l}\text { - En el ámbito empresarial las cooperativas que se } \\
\text { desarrollan en el ámbito rural, suelen no tener } \\
\text { un éxito inmediato respecto a lo financiero } \\
\text { - La adhesión libre, como uno de sus principios } \\
\text { básicos, puede ser un desestabilizador para } \\
\text { la sociedad, no todos los socios participan de } \\
\text { manera activa o encaminados hacia el bien } \\
\text { común. } \\
\text { - Existe la posibilidad de no desarrollar proyectos } \\
\text { que puedan apuntalar el desarrollo, ya que al ser } \\
\text { consensados por la comunidad y la misma pueda } \\
\text { rechazarlo por algún tipo de interés }\end{array}$ \\
\hline
\end{tabular}

Fuentes: Elaboración propia con base en Maldonado, 2009; Reyes y Romano, 2019; Quiñones, et. Al., 2019.

A partir de la tabla anterior se puede visualizar las aportaciones del cooperativismo como factor clave del turismo comunitario, señalando que la literatura científica demuestra que cuando se llevan a cabo la integración comunitaria, entre otros factores como el empoderamiento de los recursos y proyectos, hay estrategias o conductas de repercusión en el éxito de los mismos

\section{Discusión y aproximaciones}

$\mathrm{Al}$ analizar los tres conceptos enfocados al turismo, se destaca que el desarrollo local a lo largo del tiempo ha sido visualizado como una respuesta de las comunidades ante el crecimiento económico, ya que integra a los entornos de la sustentabilidad, además de verse como un proceso geográfico con variables más cuantitativas, en comparación de los esquemas de crecimiento económico surgidos a partir de la revolución industrial, donde la posesión de capital es lo más valorado, marginando al medio ambiente, la cultura, el desarrollo humano, la sustentabilidad política entre otros (Bozzi, 2001; Reyes, 2001; Barkín, 2001; Alburquerque, 2008; Quintero y Gallardo, 2008; Andrade, Marinho y Lima, 2017).

En el esquema del proceso de desarrollo local el mejoramiento de la calidad de vida es el principal indicador, pese a que el mismo no únicamente se limita a la medición numérica, sino integra un conjunto de cualidades (Boisier, 2005; Alcañiz, 2008; Juárez, 2013; Guzón Camporredondo, 2015). Sin embargo, algunos autores hacen una crítica al desarrollo local pues argumentan que no es un proceso factible en todos los tipos de territorio y en su lugar se proponen conceptos como la visión del desarrollo territorial y el endógeno (Boisier, 1999; Vázquez- Barquero, 2000; Vázquez- Barquero, 2007). 
$\mathrm{Al}$ entrelazar el desarrollo local y el turismo, se tiene que fortalecer más aún la parte integral del proceso, a pesar de que algunos autores como Sharpley (2000) Binns y Nel (2202), Borma (2015), entre otros, mencionan que el turismo es una herramienta clave en el desarrollo, especialmente aquellas modalidades que se consideran como alternativas. En el caso de Latinoamérica se pudo observar que los estudios con relación al desarrollo local y el turismo, tienen un número considerablemente más bajo en comparación del resto del mundo, en donde se emplean nuevos esquemas y modelos para ampliar el conocimiento acerca de este tipo de tópicos.

Ahora bien, una estrategia que ha sido caso de éxito para algunas localidades es el turismo de base comunitario, pues bien, la comunidad juega un papel indispensable en este desarrollo; sin embargo, ha sido fuertemente criticado debido a que no en todos los casos han sido propuestas que emanen de la comunidad, sino más bien inversiones privadas con mano de obra comunitaria (Goodwin y Santilli, 2009; De la Torre, 2010; Lucchetti y Font, 2013). Es por ello que los críticos proponen la revisión de los proyectos hacia el beneficio para la comunidad.

A partir del objetivo planteado, se vislumbra que la mayoría de los estudios demuestran que el turismo comunitario no únicamente es una herramienta de desarrollo local (Ruíz, et. Al. 2008; Burgos, 2016; Pacheco y Henríquez, 2016), sino que también es capaz de generar autoempleo, gobernanza, empoderamiento, conservación del ecosistema y englobar políticas nacionales alrededor de este tipo de turismo, generando una contribución a las actividades desarrolladas en el espacio rural (Andreu, 2008; Cardín y Álvarez, 2012 Murillo-Soto, 2016, Palomino, Gasca y López, 2016; Bravo y Zambrano, 2018)

A partir del análisis holístico, al examinar los tres conceptos de manera individual, pero sin aislarlos, entendiéndolos desde el punto de vista de múltiples interacciones y entrelazándolos de manera compleja, se puede observar en la imagen 3, que el cooperativismo puede ser un factor de desarrollo local comunitario, si se considera como una herramienta para llegar a una meta.

Imagen 3: Cooperativismo como factor clave de desarrollo local en el turismo comunitario.

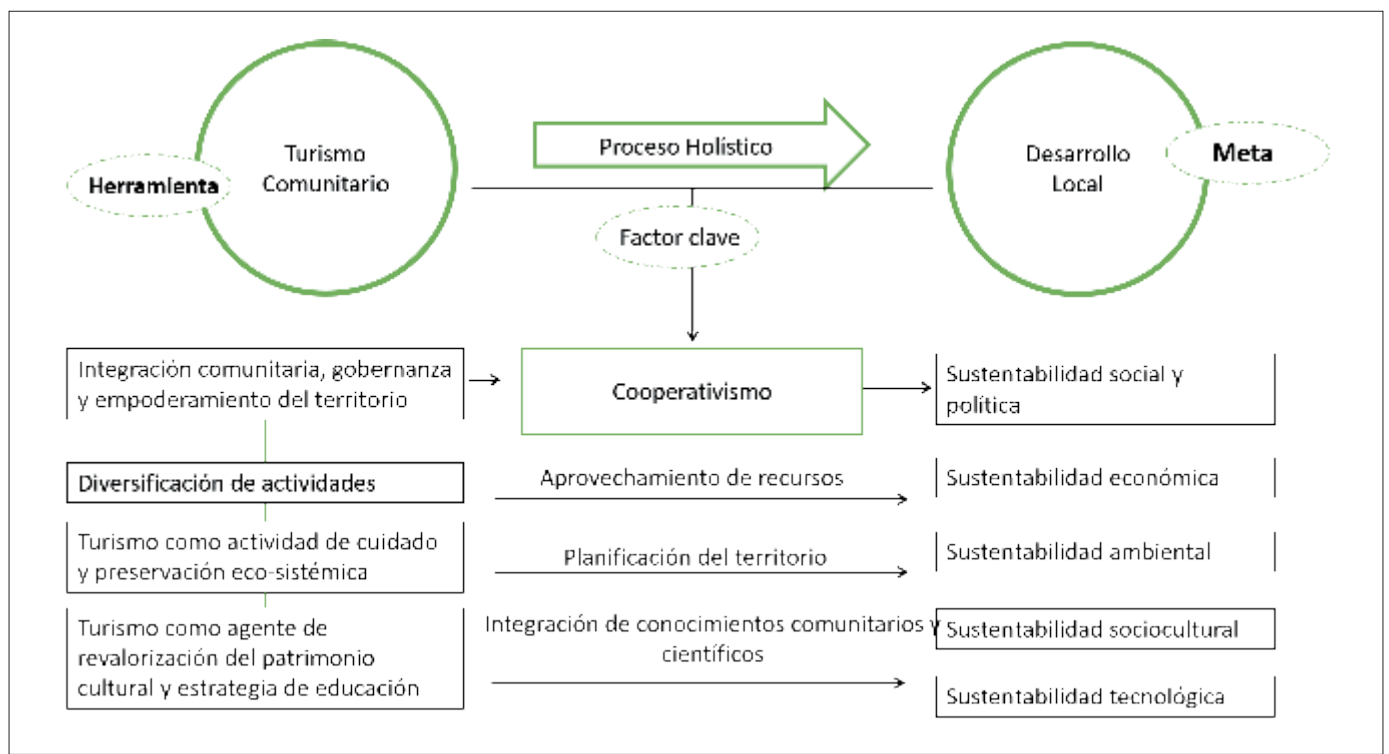

Elaboración propia con base en: Tous y Ciruela, 2005; Palomino, Gasca y López, 2016; Burgos, 2016; Mata y Pascual, 2019.

Con base en el esquema anterior, y considerando la triada de conceptos analizados, se puede identificar al cooperativismo como un factor clave del turismo comunitario y como herramienta de desarrollo local, puesto que la actividad turística con base en la comunidad, promueve la integración comunitaria, la gobernanza y el empoderamiento del territorio, con ello se busca la satisfacción del entorno socio - político del desarrollo local, siendo lo último una meta que se logra a partir de un factor clave, señalando que el cooperativismo únicamente es parte complementaria de los factores claves necesarios. 
Como se pudo demostrar mediante el estudio de la literatura científica, existen otros factores clave que deben ser tomados en cuenta en futuras investigaciones para que el proceso sea holístico y asegure una sustentabilidad como parte integral del desarrollo, como lo es la planificación del territorio, que tiene una relación estrecha con la sustentabilidad ambiental, pues en caso de no atender a los estudios de impacto ambiental, se pone entredicho la preservación del ecosistema y los recursos naturales.

\section{Consideraciones finales}

Hablar de desarrollo local es un tema complejo pues aunque queda claro que es un proceso de nivel regional, donde se involucran diversos factores, deben aprovecharse la mayor parte de los recursos disponibles, además que en algunos casos deben tener carácter innovador para poder diversificar las actividades productivas, con una visión sustentable de los recursos; es decir, aprovechar la manera en que puedan ser reparados o renovados con facilidad y así puedan continuar un proceso hacia las nuevas generaciones. El carácter endógeno del desarrollo local obliga a las comunidades a emanciparse, empoderarse y trascender como un grupo que pueda ser competitivo ante los procesos de globalización o las grandes metrópolis.

El turismo de base comunitaria es un concepto propuesto por la literatura anglosajona, y es poco utilizado en Hispanoamérica, puesto que consideran al llamado turismo comunitario como un sinónimo del concepto; sin embargo, se considera que el turismo de base comunitaria involucra una serie de elementos, donde la comunidad forma parte de la mayoría de los procesos, puestos y decisiones con respecto a los proyectos turísticos, desde la visión de un desarrollo endógeno apoyado por los gobiernos, algunos críticos sugieren que deben revisarse los proyectos de turismo de base comunitaria, pues creen que el pertenecer únicamente a una empresa que se desarrolle pero que no cuente con una estructura comunitaria, solo sería una iniciativa privada más.

Como se observó en los casos de éxito de Turismo Comunitario en América Latina (Imagen 2), este tipo de turismo es una herramienta de algunas comunidades para poder lograr un desarrollo local, ya que no solamente involucra ofrecer y vender un servicio, lugar o producto, sino también es un medio de cohesión social, educación, emancipación y promoción de una visión de sustentabilidad de los recursos. Antes de su implementación o puesta en marcha, requiere ser planificado de manera tal que las acciones, ideas o principios puedan emanar de las comunidades, siendo ellos los responsables de poder hacer trascender los proyectos. Aunado a esto los beneficios del turismo son recíprocos tanto para los visitantes, como para la comunidad; pues el visitante se lleva experiencias, así como el conocimiento que comparten las comunidades, y la comunidad puede mejorar su calidad de vida gracias a los recursos económicos que el turista deja en el lugar.

Como comentario, y propuesta final, hacia los investigadores que lleven a cabo estudios o proyectos con relación a la implementación de turismo comunitario, se considera urgente que, como contribución la literatura de desarrollo local, puedan atender los factores claves del cooperativismo y del turismo de base comunitaria, con el fin de que la toma de decisiones sea en pro de las comunidades, pero sobre todo en la revalorización de los recursos naturales y culturales que los rodean.

\section{Bibliografía}

Alburquerque, F. 2003. Teoría y práctica del enfoque del desarrollo local. Instituto de Economía y Geografía. Consejo Superior de Investigaciones Científicas, Madrid.

Alburquerque, F. 2004. El enfoque del desarrollo económico local. Serie: Desarrollo económico local y empleabilidad. Organización Internacional del Trabajo. Primera Edición. Argentina. Disponible en: https://flacsoandes.edu.ec/sites/default/files/agora/files/1251776298.area_enfoque_del_0.pdf.

Alburquerque, F. 2008. Reflexiones sobre desarrollo y territorio en América Latina. Prisma, 22, 15-34. Disponible en: https://www.delalburquerque.es/images/subidas/reflexiones.pdf

Alcañiz Moscardó, M. 2008. El desarrollo local en el contexto de la globalización. Convergencia, 15(47), 285-315.

Alvarado, J. O., y Martínez, P. N. 2013. Las teorías del desarrollo. En el análisis del turismo sustentable. Intersedes: Revista de las sedes regionales, 14(27), 144-167

Álvarez, J. y Alonso, Á. 2006. Nociones de crecimiento y desarrollo económico. Revista Galega de Economía, 12(2), 1-10. Disponible en: http://www.usc.es/econo/RGE/Vol15_2/castelan/nb1c.pdf. 
Andrade, E. Y Ochoa, M. 2017. La cooperativa como forma de organización que beneficia a todos. En: De campesinos a empresarios: Experiencia turística del Ejido "El Jorullo". Universidad de Guadalajara. Primera Edición. México. Pp. 28-47.

Andrade, J., Marinho, E. y Lima, G. 2017. Crecimiento económico y concentración del ingreso: sus efectos en la pobreza del Brasil. Revista de la CEPAL. Mo. 123. Paginas (37-57). Disponible en: https://repositorio.cepal.org/bitstream/handle/11362/42693/1/RVE123_Araujo.pdf.

Andreu, M. N. L. 2008, July. Organización y características del turismo rural comunitario en Costa Rica/Structure and characteristics of community-based rural tourism in Costa Rica. In Anales de geografía de la Universidad Complutense, 28(2), 167. Universidad Complutense de Madrid.

Arocena, J. 1997. El desarrollo local frente a la globalización. Hacia un nuevo modelo de gestión local, Municipio y Sociedad Civil en Argentina. Páginas (43-58). Disponible en: http://biblioteca.municipios. unq.edu.ar/modules/mislibros/archivos/36-Eldes.pdf.

Arocena, J. 2002. El desarrollo local: un desafio contemporáneo, $2^{a}$. Edic. Universidad Católica del Uruguay. Uruguay Marzo.

Artaraz, M. 2002. Teoría de las tres dimensiones de desarrollo sostenible. Revista Ecosistemas, 11(2).

Barkín, D. 2001. Superando el paradigma Neoliberal: desarrollo popular sustentable. En: ¿Una nueva ruralidad en América Latina? CLACSO Consejo Latinoamericano de Ciencias Sociales. Primera Edición. Buenos Aires, Argentina. Paginas (81-99) Disponible en: http://biblioteca.clacso.edu.ar/ clacso/gt/20100929012426/6barkin.pdf.

Beck, U., Moreno, B., \& Borrás, M. R. 1998. ¿Qué es la globalización?

Blázquez-Salom, M., \& Murray, I. 2011. Búnker playa-sol. Conflictos derivados de la construcción de enclaves de capital transnacional turístico español en El Caribe y Centroamérica. Scripta Nova: Revista electrónica de geografía y ciencias sociales, (15), 368.

Binns, T., \& Nel, E. 2002. Tourism as a local development strategy in South Africa. Geographical Journal, 168(3), 235-247.

Boisier, S. 1998. Post-scriptum sobre desarrollo regional: Modelos reales y modelos mentales. EURE. Vol. 24. No.72. Páginas (38).

Boisier, S. 1999. Desarrollo Local ¿De qué estamos hablando? Cámara de Comercio de Manizales Colombia. Páginas (1-28). Disponible en http://municipios.unq.edu.ar/modules/mislibros/archivos/29-DesLo.pdf

Boisier, S. 2005. ¿Hay espacio para el desarrollo local en la globalización? Revista de la CEPAL.

Borma, A. 2015. Tourism and local development. Annals of the Constantin Brancusi University of Targu Jiu Economy Series. Issue 2.

Bozzi, S. M. O. 2001. Globalización y desarrollo local: hacia una perspectiva municipalista. Revista de Estudios Sociales, (08), 21-34.

Bravo, O. y Zambrano, P. 2018. Turismo comunitario desde la perspectiva del desarrollo local: un desafío para la comuna 23 de noviembre, Ecuador. Revista Espacios. Vol.39. No.07. Paginas (28-43). Disponible en: https://www.revistaespacios.com/a18v39n07/a18v39n07p28.pdf.

Briceño, J., Cañizales, B., Rivas, Y., Lobo, H., Moreno, E., Velásquez, I., \& Ruzza, I. 2010. La holística y su articulación con la generación de teorías. Educere, 14(48), 73-83.

Buendía-Martínez, I. y Cóte, A. 2014. Desarrollo territorial rural y cooperativas: un análisis desde las políticas públicas. Cuadernos de Desarrollo Rural. No. 11. Paginas (35-54). Disponible en: http:// www.scielo.org.co/pdf/cudr/v11n74/v11n74a03.pdf.

Burgos, R. 2016. El turismo comunitario como iniciativa de desarrollo local. Caso de las localidades de Ciudad Bolívar y Usme zona rural de Bogotá. Revista Hallazgos. No. 26. Paginas (193-214). Disponible en: http://www.scielo.org.co/pdf/hall/v13n26/v13n26a09.pdf

Cañada, E. 2015. La comercialización del turismo comunitario en América Latina. Anuario de Estudios Centroamericanos, 159-189.

Cardín, M. y Álvarez, C. 2012. El turismo rural comunitario en costa rica. En XI Congreso Internacional de Ingeniería de Proyectos Lugo, 26-28 de septiembre 2012. Pp. 1658-1666.

Castro-Gómez, S. 1998. Latinoamericanismo, modernidad, globalización. Prolegómenos a una crítica poscolonial de la razón. Teorías sin disciplina. Latinoamericanismo, poscolonialidad y globalización en debate, 169-205.

Cesar, A., Arnaiz, S. y Anaya, L. 2016. Turismo: modelo sostén en el capitalismo global. En Los Retos del Turismo. Universidad de Guadalajara. Centro Universitario de la Costa. Primera Edición. México. Páginas (11-29). Disponible en http://www.cuc.udg.mx/sites/default/files/publicaciones/2016\%20-\%20 Los\%20retos\%20del\%20turismo.pdf. 
De la Torre, G. M. V., Guzmán, T. J. L. G., \& Caridad, J. M. 2007. Turismo comunitario en Centroamérica. Un análisis econométrico. Papers de Turisme, (41), 57-73.

De la Torre, S. 2010. Turismo comunitario, ¿otro sueño inalcanzable? Polémika, 2(5).

Di Pietro, L. 2001. Hacia un desarrollo integrador y equitativo: una introducción al desarrollo local. Ediciones CICCUS-La Crujía. Páginas (111- 236). Disponible en: http://www.bibliotecavirtual.info/ wp-content/uploads/2011/06/hacia_un_desarrollo_integrador_y_equitativo.pdf.

Díaz, C. 2010. Ecoturismo comunitario y Género en la Reserva de la Biósfera de Los Tuxtlas. Pasos. Revista de Turismo y Patrimonio Cultural, 8(1), 151-165. Obtenido de http://www.pasosonline.org/ Publicados/8110/PASOS19.pdf\#page $=159$

Goodwin, H. y Santilli, R. 2009. Community- Based Tourism: a Success? ICTR Occasional Paper. 11. Páginas (37). Disponible en: http://www.andamandiscoveries.com/press/press-harold-goodwin.pdf.

Gruter, M. 2013. Impactos negativos del turismo. Torres Lezama, VicenteiAraujo, Edward Pierre (comp.) Antropología del Turismo. La industria sin chimeneas. Cuzco: Tinkuy, 63-70.

Guzón Camporredondo, A. (2015). Desarrollo local en Cuba: retos y perspectivas. CIPS.

Herrera, S. B. 1997. Globalización y desarrollo mundial. Editorial de Ciencias Sociales.

Hernández Vaca, J. 2005. La administración comunitaria del pueblo azteca. Estudios políticos (México), (6), 113-144.

Instituto Costarricense de Turismo. 2006. Plan Nacional de Desarrollo Turístico de Costa Rica 2002-2012. Actualización 2006. Costa Rica.

Jafari, J. 2005. El turismo como una disciplina científica. Revista Política y Sociedad. Vol. 42. No. 1. Páginas (39-32).

Juárez, G. 2013. Revisión del concepto de desarrollo local desde una perspectiva territorial. Revista Líder. Vol. 23. Páginas (9-28). Disponible en: http://ceder.ulagos.cl/lider/images/numeros/23/1.-LIDER\%20 23_Juarez_pp9_28.pdf.

Lalangui, J., Espinoza, C. y Pérez, M. 2017. Turismo sostenible. Un aporte a la responsabilidad social empresarial: Sus inicios, características y desarrollo. Universidad y Sociedad Revista Científica de la Universidad de Cienfuegos. Vol. 9. No.1. Páginas (148-153). Disponible en: http://scielo.sld.cu/pdf/ rus/v9n1/rus21117.pdf

López-Guzmán, T., \& Sánchez-Cañizares, S. 2009. Turismo comunitario y generación de riqueza en países en vías de desarrollo. Un estudio de caso en El Salvador. REVESCO: revista de estudios cooperativos, (99), 85-103.

López-Guzmán, T., Sánchez-Cañizares, S., \& Pavón, V. 2011. Community-based tourism in developing countries: a case study. Tourismos, 6(1).

Lorenzo, A. M. C., \& Zamora, D. T. 2005. Cooperativas agrarias turísticas. Hacia un modelo innovador y sostenible de gestión rural. REVESCO. Revista de Estudios Cooperativos, (86), 39-60.

Lucchetti, V. G., \& Font, X. 2013. Community based tourism: Critical success factors. ICRT occasional paper, 27, 1-20.

Maldonado, C. 2005. Pautas metodológicas para el análisis de experiencias de turismo comunitario. Serie Red de Turismo Sostenible. No. 73. Comunitario para América Latina. Organización Mundial del Trabajo. Primera Edición. Ginebra, Suiza.

Maldonado, C. 2009. Fortaleciendo redes de turismo comunitario REDTURS en Latinoamérica. Revista @local.glob. No. 4. Paginas (8-14).

Maldonado, M. y Maldonado, C. 2019. Empresas Cooperativas en la Actividad Turística sustentable de México. Revista InterSedes. Vol. 20. Paginas (12)

Maraña, M. 2010. Cultura y desarrollo: Evolución y perspectivas. UNESCO- Etxea Cuadernos de Trabajo. No. 1 Paginas (1-30). Disponible en: http://www.unescoetxea.org/dokumentuak/Cultura_desarrollo.pdf.

Martín-Barbero, J. 2003. La globalización en clave cultural. Una mirada latinoamericana.

Martínez, I. B. 1999. Desarrollo rural en la Unión Europea: el programa LEADER y el papel de las sociedades cooperativas. Cuadernos de desarrollo rural= International journal of rural development, (42), 35-54.

Mata, E. y Pascual A. 2019. Desarrollo local y ecoturismo en Ayutla, Querétaro, México. El cooperativismo como estrategia de desarrollo: Caso Cooperativa Ecoturística "El paraíso". Tesis de Licenciatura en Turismo. Universidad Autónoma del Estado de México.

Mateus, J. R., \& Brasset, D. W. 2002. La globalización: sus efectos y bondades. Economía y desarrollo, $1(1), 65-77$. 
Mendoza, O., Figueroa, H., \& Godínez, M. 2015. Turismo comunitario pro-pobre en el ejido El Rosario, Reserva de la Biosfera de la mariposa Monarca. El periplo sustentable (29), 92-119. Obtenido de https://www.redalyc.org/pdf/1934/193440418006.pdf

MINCETUR 2015. Turismo rural comunitario. Sitio Web. https://www.mincetur.gob.pe/producto-turistico/ turismo-rural-comunitario/. Consultado el: 06/Diciembre/2019.

Monge- Rodríguez, V. y Macías- Chávez, A. 2016. El desarrollo local. Enfoques para su conceptualización. OLIMPIA Revista de la Facultad de Cultura Física de la Universidad de Granma. Vol. 13. No.39. Paginas (225-238). Disponible en: http://.Dialnet-ElDesarrolloLocalEnfoquesParaSuConceptualiza cion-6210548\%20(1).pdf

Montoya, M. 2013. Turismo Comunitario y desarrollo rural: Interacción y escalonamiento de innovaciones. Estudios sobre el desarrollo. No. 6. Instituto de Estudios peruanos. Lima, Perú.

Murillo-Soto, V. 2016. Redes de Turismo Rural Comunitario: La experiencia de Argentina y Costa Rica. Revista Tecnología en Marcha, 29, 59-68.

Orgaz, F. 2013. El turismo comunitario como herramienta para el desarrollo sostenible de destinos subdesarrollados. Revista Nómadas. Vol. 38. Páginas 14

Ortiz, D., Chávez R. Y Osmin, J. 2017. Sustentabilidad y contribuciones para el desarrollo local. En: De campesinos a empresarios: Experiencia turística del Ejido "El Jorullo". Universidad de Guadalajara. Primera Edición. México. Paginas (139-152).

Osorio García, M. 2010. Turismo masivo y alternativo. Distinciones de la sociedad moderna/posmoderna. Convergencia, 17(52), 235-260.

Pacheco, G. y Henríquez, C. 2016. El turismo de base comunitaria y los procesos de gobernanza en la comuna de Panguipulli, Sur de Chile. Revista Gestión Turística. No. 25. Páginas (42-62).

Palacios, C. G. 2016. Turismo comunitario en Ecuador: ¿quo vadis? Estudios y perspectivas en turismo, 25(4), 597-614.

Palomino, B., Gasca, J. y López, G. 2016. El Turismo comunitario en la Sierra Norte de Oaxaca perspectiva desde las instituciones y la gobernanza en territorios indígenas. Revista El Periplo Sustentable Universidad Autónoma del Estado de México. Núm. 30. Páginas (06-37)

Palmas, D., Serrano- Barquín, R. y Osorio, M. 2014. "Xíinbal Tuukul Ya'Ax", Revista Turydes: Turismo y Desarrollo, n. 17 (diciembre 2014). En línea: http://www.eumed.net/rev/turydes/17/xiinbal.html

Pastor, V. J., Jurado, C. C., \& Domingo, A. S. 2011. Desarrollo rural a través del turismo comunitario. Análisis del valle y cañón de colca. Gestión turística, (15), 1-20.

Pearce, D. G. 1992. Alternative tourism: Concepts, classifications, and questions. Tourism alternatives: Potentials and problems in the development of tourism, 15-30.

Pérez Morfi, D., Nuñez Paula, I., \& Font Graupera, E. 2016. Globalización y desarrollo local, una propuesta metodológica de gestión de información y el conocimiento. Economía y Desarrollo, 157(2), 107-119.

Picornell, C. 2015. Los impactos del turismo. Papers de turisme, (11), 65-91.

Pilquiman, M. 2016. El turismo comunitario como una estrategia de supervivencia. Resistencia y reivindicación cultural indígena de comunidades mapuche en la Región de los Ríos (Chile). Revista Estudios y Perspectivas del Turismo. Vol. 25. Núm.4. Páginas (439-459).

PNUD. 1990. Desarrollo humano informe 1990. Tercer Mundo Editores. Colombia. Paginas (220). Disponible en: http://hdr.undp.org/sites/default/files/hdr_1990_es_completo_nostats.pdf.

PNUD 2012. Federación Plurinacional de Turismo Comunitario (FEPTCE). Estudios de caso de la iniciativa ecuatorial. Soluciones locales de desarrollo sostenible para las personas, la naturaleza y las comunidades resilientes. Serie de Estudios de caso PNUD Iniciativa Ecuatorial. Páginas (9). Disponible en https://www.equatorinitiative.org/wp-content/uploads/2017/05/case_1_1363900152.pdf.

Prats, L. 2012. Las Cooperativas y su influencia en la capacidad de innovación de las destinaciones turísticas. Revista Cooperativismo y Desarrollo. Vol. 20.

Prieto, J. M. L. 2002. Guerras mundiales y globalización. Ábaco, 32(33), 127-139.

Quintero, J. 2008). Turismo y desarrollo local en México. El caso del municipio de Cabo Corrientes en Jalisco. Guadalajara: Universidad de Guadalajara, Universidad Complutense de Madrid.

Quiñones, Y. G., Castrejón, Y. D. P., Martínez, E. E. V., \& Barquín, R. S. 2019. Una red de cooperación empresarial para el desarrollo local corredor turístico "Las Truchas"-Malinalco, México. Estudios y Perspectivas en Turismo, 28(1), 207-225.

Reyes, G. E. 2001. Principales teorías sobre el desarrollo económico y social. Nómadas. Critical Journal of Social and Juridical Sciences, (4). Disponible en: https://www.redalyc.org/pdf/181/18100408.pdf

Reyes, J. y Romano, G. 2019. Cooperativas Turísticas del norte de Quintana Ro: ¿Procesos de sustentabilidad turística? TURyDES Revista de Turismo y Desarrollo Local. Vol. 12. Paginas (12). 
Reyes, M. Ortega, A. y Machado, L. 2017. Modelo para la gestión integrada del turismo comunitario en Ecuador, caso de estudio Pastaza. REVESCO. No.123. Paginas (250-275). Disponible en https:// revistas.ucm.es/index.php/REVE/article/view/53242 .

Rubio, C. H. 2002. La teoría del crecimiento endógeno y el comercio internacional. Cuadernos de estudios empresariales, (12), 95.

Sánchez, P. Y Prada, A. 2015. Del concepto de crecimiento económico al de desarrollo de las naciones: una aplicación a la Unión Europea. Revista de Economía Mundial. No. 40. Paginas (221-251). Disponible en: https://www.redalyc.org/pdf/866/86641407010.pdf.

Sharpley, R. 2000. Tourism and sustainable development: Exploring the theoretical divide. Journal of Sustainable tourism, 8(1), 1-19.

Serrano-Barquín, R. 2006. Tesis Doctoral Desarrollo, Sustentabilidad y Turismo en una comunidad lacustre del Valle de Toluca, Caso: San Miguel Almaya, Estado de México. Toluca, México: UAEM.

Skewes, J., Henríquez, C. y Pilquiman, M. 2012. Turismo comunitario o de base comunitaria: una experiencia alternativa de hospitalidad vivida en el mundo Mapuche. Tlalcao sur de Chile. CULTUR Revista de Cultura y Turismo. Año 6. No.2. Páginas (73-85) Disponible en: Dialnet-TurismoComun itarioODeBaseComunitaria-5315656\%20(1).pdf

Tello, M. D. 2006. Las teorías del desarrollo económico local y la teoría y práctica del proceso de descentralización en los países en desarrollo. Pontificia Universidad Católica del Perú, Departamento de Economía. Disponible en: https://www.researchgate.net/profile/Mario_Tello/publication/46456209_ Las_teorias_del_desarrollo_economico_local_y_la_teoria_y_practica_del_proceso_de_descentralizacion_en_los_paises_en_desarrollo/links/09e41506aebe11855f000000/Las-teorias-del-desarrollo-economico-local-y-la-teoria-y-practica-del-proceso-de-descentralizacion-en-los-paises-en-desarrollo.pdf

Toselli, C. 2015. Turismo, planificación estratégica y desarrollo local. TURyDES Revista de Turismo y Desarrollo Local. Vol. 8, No. 18. Paginas (8).

Toselli, C. 2019. Turismo, patrimonio cultural y desarrollo local. Evaluación del potencial turístico de Aldeas Rurales en la Provincia de Entre Ríos, Argentina.

Tous Zamora, D., \& Ciruela Lorenzo, A. M. 2005. Responsabilidad social y cultura en las sociedades cooperativas agrarias. Modelo general de balance social. CIRIEC-España, Revista de Economía Pública, Social y Cooperativa, (53), 209-240.

Valle, P. E., \& Cariño, O. 2009. Desarrollo del Turismo Comunitario en la Laguna San Ignacio, Baja California Sur. En D. Chávez, R. E. Andrade, \& S. Espinoza, Turismo Comunitario en México (págs. 34-46). Puerto Vallarta, Jalisco: Universidad de Guadalajara. Obtenido de http://udgart.com/assets/ doc/Turismo-comunitario-en-mexico.pdf

Vázquez- Barquero, A. 2000. Desarrollo económico local y descentralización: aproximación a un marco conceptual. Proyecto CEPAL/GTZ "Desarrollo económico local y descentralización en América Latina”. Edición CEPAL. Santiago, Chile. Disponible en: https://repositorio.cepal.org/bitstream/ handle/11362/31392/S00020088_es.pdf.

Vázquez-Barquero, A. 2007. Desarrollo endógeno. Teorías y políticas de desarrollo territorial. Revista investigaciones regionales. No. 11. Paginas (183-210). Disponible en: https://www.redalyc.org/ pdf/289/28901109.pdf.

Vega, R. A., y Vivas, X. M. 2007. El turismo como motor de crecimiento económico. Anuario jurídico y económico escurialense, (40), 677-710. 